\title{
Inventário florístico florestal de Santa Catarina: espécies da Floresta Ombrófila Mista
}

Flora of the mixed ombrophyllous forest in Santa Catarina state, according of the forest and floristic inventory of Santa Catarina

\author{
André Luís de Gasper ${ }^{1,5}$, Lucia Sevegnani ${ }^{1}$, Alexander Christian Vibrans ${ }^{2}$, Marcos Sobral ${ }^{3}$, \\ Alexandre Uhlmann ${ }^{4}$, Débora Vanessa Lingner ${ }^{2}$, Morilo José Rigon-Júnior ${ }^{1}$, Marcio Verdi ${ }^{2}$, \\ Anita Stival-Santos ${ }^{2}$, Susana Dreveck ${ }^{2} \&$ Alexandre Korte ${ }^{2}$
}

\begin{abstract}
Resumo
Este estudo é resultado da amostragem sistemática da flora da floresta ombrófila mista em Santa Catarina, realizada em 155 pontos amostrais em toda a sua extensão e permite atualizar o conhecimento sobre a ocorrência de espécies. Foram registradas 925 espécies de espermatófitas, distribuídas em 439 gêneros e 116 famílias botânicas. A família com a maior riqueza específica foi Asteraceae (119 espécies), seguida por Myrtaceae (88), Fabaceae (58) e Solanaceae (52). Dentre as famílias restantes, 34 apresentaram somente uma e outras 27 tiveram duas espécies registradas. Os gêneros com maior número de espécies foram Solanum (31 espécies), Baccharis (27), Eugenia (23), Ocotea (21) e Myrcia (19). Araucaria angustifolia (Bertol.) Kuntze, Butia eriospatha (Mart. ex Drude) Becc., Ocotea odorifera (Vell.) Rohwer e O. porosa (Nees \& Mart.) Barroso são as espécies ameaçadas de extinção registradas. Entre todas as espécies do componente arbóreo ou arbustivo/subarbóreo, 56,5\% são comuns a ambos os componentes e dentre as 194 espécies arbóreas citadas para o planalto catarinense, 157 foram amostradas pelo IFFSC. O levantamento florístico extra registrou 474 espécies de angiospermas a mais do que o levantamento nas unidades amostrais do IFFSC. O IFFSC amostrou um conjunto significativo das espécies do Domínio Floresta Atlântica. Estas coletas georreferenciadas e realizadas com uma amostragem sistemática e consistente, representam um importante avanço e atualização do conhecimento da flora de Santa Catarina. Inventários sistemáticos desta natureza são necessários às demais regiões no Sul do Brasil, para que se possa compor um banco de dados consistente e atualizado e possibilitar a implantação de políticas de conservação e manejo.
\end{abstract}

Palavras-chave: coleta botânica, diversidade, Floresta de Araucária, lista de espécies, Mata Atlântica.

\begin{abstract}
This study is based on a systematic sample of the flora of mixed ombrophilous forest in Santa Catarina, composed by 155 sample plots located all over it's geographic extension. We recorded 925 spermatophytes species divided in 439 genera and 116 botanical families. The family with major specific richness was Asteraceae ( 119 species), followed by Myrtaceae (88), Fabaceae (58) and Solanaceae (52). Among the remaining families, 34 of them had only one species and other 27 families obtained only two registered species. The genera with major number of species were: Solanum (31 species), Baccharis (27), Eugenia (23), Ocotea (21), and Myrcia (19). Araucaria angustifolia, Butia eriospatha, Ocotea odorifera e O. porosa are redlist species. Among all the species of the arboreous and shrubby/subarboreous component, $56.5 \%$ are common in both components. Among the 194 tree species reported in the literature quoted for the altiplano of Santa Catarina, 157 were registered in the present study. The floristic survey in the surroundings of the IFFSC sample plots recorded another 474 angiosperm species, besides the species collected within the sample plots. The IFFSC sampled a significant number of Atlantic Forest species, held in a georeferred collection that represents an important progress for the knowledge about the flora of Santa Catarina. Systematics floristic inventories are necessary also in other regions in southern of Brazil to make a consistent and updated database.

Key words: botanical collection, diversity, Araucaria forest, species list, Atlantic Forest.
\end{abstract}

Este artigo possui material adicional em sua versão eletrônica.

\footnotetext{
${ }^{1}$ Universidade Regional de Blumenau, Herbário Dr. Roberto Miguel Klein, R. Antônio da Veiga 140, Victor Konder, 89012-900, Blumenau, SC, Brasil.

${ }^{2}$ Universidade Regional de Blumenau, Depto. Engenharia Florestal, R. São Paulo 3250, Itoupava Seca, 89030-000, Blumenau, SC, Brasil.

${ }^{3}$ Universidade Federal de São João Del-Rei, Pça. Frei Orlando 170, 36307-352, São João Del-Rei, MG, Brasil.

${ }^{4}$ Embrapa Florestas, Estrada da Ribeira, km 111, C.P. 319, 83411-000, Colombo, PR, Brasil.

${ }^{5}$ Autor para correspondência: algasper@gmail.com
} 


\section{Introdução}

Recentes convenções e acordos internacionais (Canhos et al. 2004) postulam que seus signatários formulem suas listas de espécies, para conhecer sua biodiversidade. Contudo, coletas de plantas estão geralmente associadas à regiões próximas a grandes centros de pesquisa e universidades (Schatz 2002; Sobral \& Stehmann 2009), constituindo o chamado de efeito-museu (Hopkins 2007) e, em outros casos, os dados disponíveis são em geral de baixa qualidade (Hortal et al. 2007).

A disponibilização de dados de coleta em grandes bancos de dados como o do INCT Herbário Virtual da Flora e dos Fungos (INCT 2012), tem possibilitado a realização de estudos que envolvem grandes análises em níveis global (Kreft \& Jetz 2007; Yesson et al. 2007), regional (Werneck 2011) e local (Gasper 2012). Além disso, a disponibilização de informações ambientais como as de Hijmans et al. (2005) permitem trabalhos com os de modelagem de espécies de forma cada vez mais precisa (p.ex., Hernandez et al. 2008). Contudo, para que isso seja possível, coletas intensificadas e padronizadas precisam ser efetuadas. Neste sentido, o estado de Santa Catarina, através do Inventário Florístico Florestal (IFFSC), tem contribuído sobremaneira para melhorar a qualidade dos dados, disponibilizando o material fértil para consulta no herbário Dr. Roberto Miguel Klein (FURB).

Dentre as regiões fitoecológicas (aqui consideradas as propostas por Leite 2002) com maior pressão antrópica, e sendo a de maior cobertura florestal em Santa Catarina, a Floresta Ombrófila Mista (FOM) cobre menos de $25 \%$ da sua área original (Leite \& Klein 1990; Vibrans et al. 2013), o que ilustra o elevado grau de alteração de sua cobertura original. Sua redução e descaracterização resultaram da intensa exploração madeireira, bem como da conversão das áreas florestais em áreas de agricultura e pecuária (Nascimento et al. 2001; Sampaio \& Guarino 2007; Vibrans et al. 2011), além da contínua exploração, embora em escala decrescente, dos fragmentos remanescentes.

Diante deste cenário, o conhecimento sobre a flora da FOM é imprescindível, não apenas para seu diagnóstico, mas também para direcionar estratégias de conservação (Rivers et al. 2010). Nessa perspectiva, os estudos conduzidos por Klein $(1960 ; 1963 ; 1984)$ e os da Flora Ilustrada Catarinense (Reitz 1965) contribuíram muito para o conhecimento das espécies que compõem a FOM. Entretanto, a maioria dos estudos realizados na última década é restrita à análise do componente arbóreo da floresta (Nascimento et al. 2001; Randon Neto et al. 2002; Cordeiro \& Rodrigues 2007; Lingner et al. 2007; Klauberg et al. 2010), sendo raros estudos do componente herbáceo (CitadiniZanete et al. 2011), embora forneçam informações relevantes no estudo de comunidades florestais, principalmente relacionadas à compreensão dos mecanismos de regeneração da floresta.

Diante do exposto, fica evidente a necessidade de estudos que contemplem todo o espectro de formas de vida abrigadas na FOM. Inventários florísticos e florestais têm assumido um papel fundamental, principalmente quando o material botânico é depositado em coleções facilmente acessíveis (Guedes-Bruni et al. 2002; Corona et al. 2011). O Inventário Florístico Florestal de Santa Catarina IFFSC (Vibrans et al. 2010) realizou um importante levantamento quali-quantitativo da FOM, entre 2008 e 2010, que representa um excelente banco de dados para a pesquisa do estado atual da flora, devido à sua abrangência territorial e ao seu método de amostragem sistemática e uniforme.

Com base nos dados levantados pelo IFFSC, o presente estudo tem por objetivo caracterizar a composição florística atual da FOM catarinense, contrapondo-a a dados históricos (Reitz 1965), além de disponibilizar os dados de forma pública e possibilitar aos pesquisadores o conhecimento do registro de ocorrência das espécies nesta região fitoecológica.

\section{Material e Métodos}

A área original de cobertura da FOM em Santa Catarina abrange $42.851 \mathrm{~km}^{2}$, equivalente a 44,94\% do território estadual (Klein 1978), ocorrendo predominantemente em áreas situadas entre 500 e $1.500 \mathrm{~m}$ de altitude (Backes 2009). Está sob clima Mesotérmico ( $\mathrm{Cfb}$ ), com chuvas regularmente distribuídas ao longo do ano, sem estação seca e com até 30 dias de geadas por ano, havendo regiões onde neve pode ocorrer em até quatro dias em um ano (Backes 2009).

Os dados apresentados neste estudo foram coletados durante a execução do Inventário Florístico Florestal de Santa Catarina (IFFSC). A sua metodologia detalhada está descrita em Vibrans et al. (2010), que em síntese é constituída por uma amostragem com unidades amostrais (UA) instaladas em remanescentes florestais de forma sistemática, nas interseções de uma grade de 10 $\times 10 \mathrm{~km}$, abrangendo todo território catarinense. Cada unidade amostral é composta por quatro subunidades de $20 \times 50 \mathrm{~m}$, localizadas na direção 
dos quatro pontos cardeais, distando $30 \mathrm{~m}$ do ponto central, totalizando uma área amostral de $4.000 \mathrm{~m}^{2}$.

Neste estudo foram analisados os dados do conjunto de espécies coletadas em 155 remanescentes amostrados, localizados em altitudes entre 514 e $1.560 \mathrm{~m}$, sendo 143 na referida grade e 12 fora dela, mas em remanescentes considerados em melhor estado de conservação (Fig. 1). As coletas foram efetuadas de forma estratificada: o componente arbóreo (indivíduos com DAP $\geq 10 \mathrm{~cm}$ ) foi amostrado na totalidade da UA; o componente arbustivo/subarbóreo (indivíduos com DAP $\leq 10 \mathrm{~cm}$ e altura total $\geq 1,50 \mathrm{~m}$ ) foi amostrado em subparcelas de $100 \mathrm{~m}^{2}$. Além disso, buscou-se coletar todo material fértil ao alcance das equipes de campo, dentro e nas proximidades das UAs, sem obediência aos critérios de tamanho, no denominado "levantamento florístico extra" (Vibrans et al. 2010). Apenas as espécies de gimnospermas e angiospermas (espermatófitas) nativas foram analisadas neste estudo.

A identificação do material foi realizada em campo, ou então em laboratório, através de bibliografia e comparações com a coleção do herbário
Dr. Roberto Miguel Klein (FURB), ou consultandose especialistas. Todo o material botânico fértil coletado foi depositado no herbário FURB da Fundação Universidade Regional de Blumenau, sendo georreferenciado na origem, e doações foram distribuídas principalmente para os herbários BHCB, MBM, R, ICN e RB (siglas de acordo com Thiers 2012). Os espécimes testemunho apresentados neste trabalho referem-se sempre ao material fértil coletado pelo IFFSC, em Santa Catarina. Todas as coletas podem ser consultadas na base de dados do INCT Herbário Virtual (FURB 2012).

A classificação das famílias de angiospermas segue APG III (2009) e para as gimnospermas Christenhusz et al. (2011). Com o intuito de evitar superestimativas, apenas as coletas identificadas até espécie foram contabilizadas, exceto algum gênero, caso nenhuma espécie do mesmo tenha sido identificada na área de estudo.

Com base nos registros das espécies, foi elaborado um mapa de intensidade amostral e riqueza acumulada de táxons, em quadrículas de $20 \times 20 \mathrm{~km}$, usando o software ArcGis 10 (ESRI 2011).

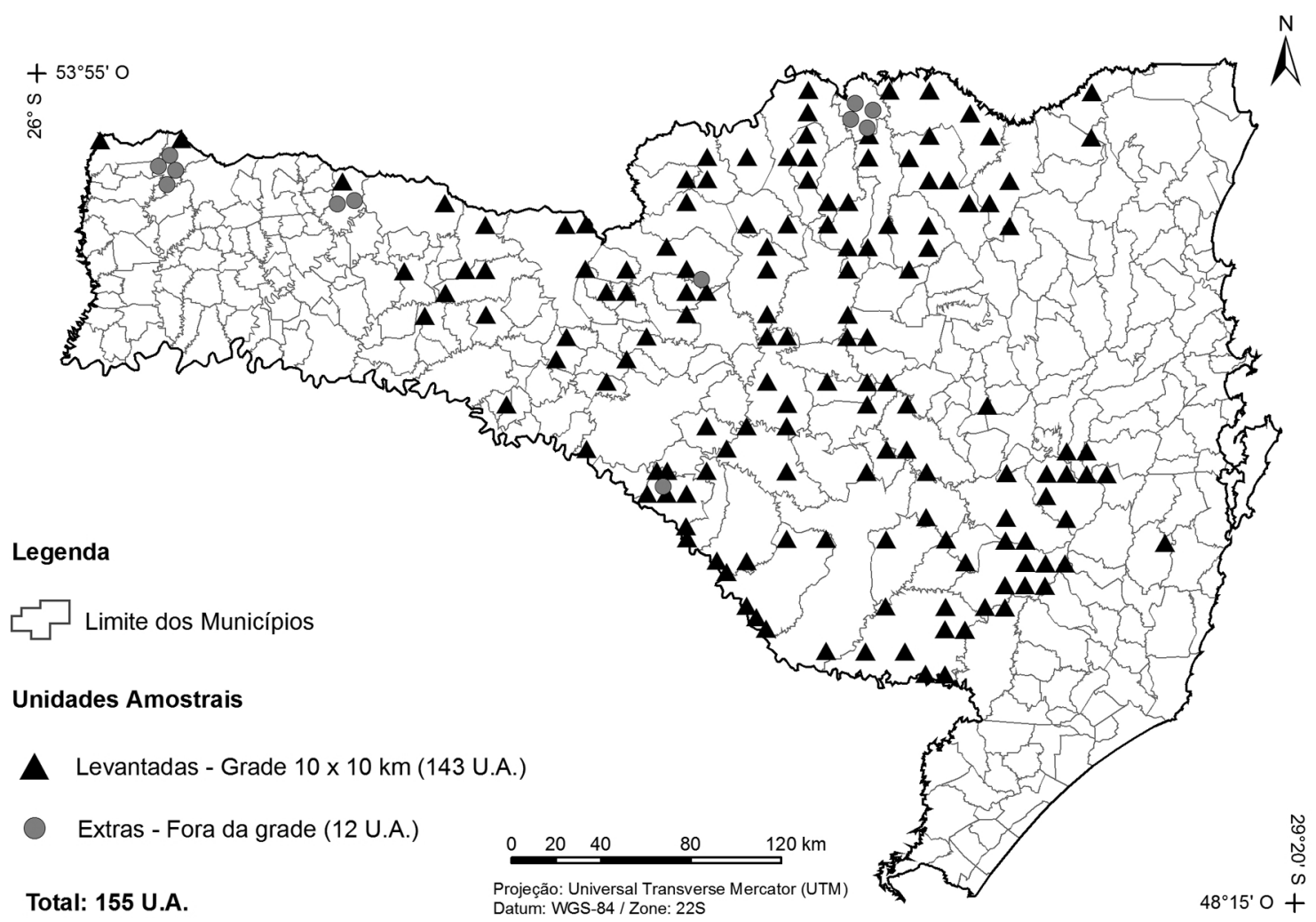

Figura 1 - Localização das 155 unidades amostrais inventariadas na floresta ombrófila mista em Santa Catarina, Brasil. Figure 1 - Localization of 155 sample plots within mixed ombrophilous forest in Santa Catarina State, Brazil. 


\section{Resultados}

Considerando todos os componentes abrangidos pelo presente estudo, foram registradas 925 espécies, sendo três gimnospermas (Araucaria angustifolia, Podocarpus lambertii e P. sellowii) e 922 angiospermas (ver Apêndice na versão on line deste trabalho), distribuídas em 439 gêneros e 116 famílias botânicas. A família com a maior riqueza específica foi Asteraceae (119 espécies), seguida por Myrtaceae (88), Fabaceae (58), Solanaceae (52), Melastomataceae (43), Lauraceae (39), Orchidaceae (37), Rubiaceae (32), Poaceae (28) e Cyperaceae e Piperaceae, cada qual com 23 espécies (Fig. 2). Dentre as famílias restantes, 34 apresentaram somente uma espécie registrada e outras 27 tiveram duas (Tab. 1). Os gêneros com maior número de espécies foram Solanum (31 espécies), Baccharis (27), Eugenia (23), Ocotea (21), Myrcia (19), Leandra (18), Peperomia (15) e Myrceugenia (15).

Entre as 925 espécies registradas, 450 delas foram encontradas nos componentes arbóreo e arbustivo/subarbóreo, sendo 56,5\% (256 espécies) comuns a ambos. Das 194 espécies restantes, 110 foram registradas exclusivamente no componente arbóreo e 84 exclusivamente no arbustivo/ subarbóreo. Com relação às espécies do componente arbóreo, ressaltam-se algumas cujos regenerantes não foram registrados: Trithrinax brasiliensis, Alchornea sidifolia, Albizia niopoides, Apuleia leiocarpa, Calliandra foliolosa, Ocotea daphnifolia, Ocotea floribunda e Balfourodendron riedelianum.

No levantamento florístico extra, foram registradas 770 espécies, sendo 475 espécies exclusivas deste levantamento. Das 770 espécies, 354 são árvores e arbustos, 197 ervas terrícolas,

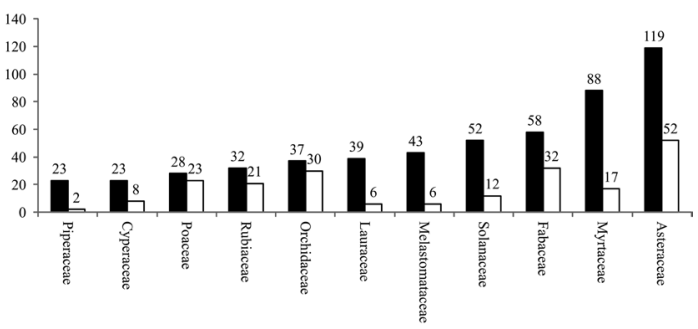

Figura 2 - Síntese do número de espécies e gêneros das principais famílias registradas na Floresta Ombrófila Mista. Barras pretas correspondem às espécies e barras brancas aos gêneros.

Figure 2 - Synthesis of species and genera of the main families collected on Mixed Ombrophylous Forest. Black bars correspond to the species and white bars to the genera.
70 epífitos e 58 lianas (em alguns casos a mesma espécie pode ter sido coletada como epífita ou erva terrícola, por exemplo, sendo contada nas duas classificações). Do total de espécies, quatro estão na lista daquelas ameaçadas de extinção (MMA 2008): Araucaria angustifolia, Butia eriospatha, Ocotea odorifera e O. porosa.

Com relação ao mapa de intensidade amostral e riqueza (Fig. 3), baseado no registro das espécies nas áreas abrangidas pelo estudo, foi obtida uma riqueza média de aproximadamente 100 espécies em cada quadrícula de $400 \mathrm{~km}^{2}$.

\section{Discussão}

Os resultados obtidos através da análise dos componentes florísticos integrantes deste estudo propiciaram o registro de $22,7 \%$ das 4.116 espécies citadas na Flora do Brasil, com ocorrência em Santa Catarina (Forzza et al. 2010). Sabe-se, contudo, que a maior riqueza de espécies no estado concentrase na região da Floresta Ombrófila Densa, que se distribui principalmente pelas encostas das Serras Geral e do Mar, com grande endemismo e riqueza de espécies (Martinelli 2007). Considerando o estudo de Stehmann et al. (2009), o presente estudo registrou $43 \%$ de todas as espécies brasileiras citadas para esta região fitoecológica.

Sob uma perspectiva geral, os resultados obtidos demonstram que a maior riqueza de espécies ocorre nas principais famílias botânicas já apontadas por Stehmann et al. (2009) e por diversos estudos fitossociológicos e florísticos em Santa Catarina (Reitz et al. 1978; Negrelle \& Silva 1992; Formento et al. 2004), com destaque para as famílias Myrtaceae e Lauraceae nas áreas de FOM no planalto catarinense (Klauberg et al. 2010).

$\mathrm{O}$ fato de Asteraceae ter sido a família mais rica neste estudo pode ser explicado pelo caráter pioneiro de suas espécies e pelo contato da FOM com os campos sulinos, o que facilita o estabelecimento destas nas áreas de floresta (Pillar et al. 2009). Aliado a isso, o atual estado de degradação da FOM, com predominância de ambientes abertos, possivelmente contribui para a distribuição e abundância destas espécies em Santa Catarina.

Dentre os gêneros com maior riqueza, ressaltase a importância de Myrceugenia (15 espécies) na composição florística da FOM em Santa Catarina. Das 30 espécies citadas para todo o país, metade foi registrada neste estudo, fazendo com que a área de estudo seja considerada um centro de endemismo para o gênero (Sobral 2003; Landrum 1981). 
Tabela 1 - Síntese da flora da Floresta Ombrófila Mista e seus ecótonos com a Floresta Estacional Decidual e Estepe Ombrófila, amostrada pelo Inventário Florístico Florestal de Santa Catarina.

Table 1 - Synthesis of flora of Mixed Ombrophylous Forest including ecotones with Seasonal Deciduous Forest and "Campos Naturais", as sampled by IFFSC.

Florística do Inventário Florestal de Santa Catarina $n^{0}$ espécies

Total de espécies amostradas

Famílias de angiospermas

Famílias com 10 ou mais espécies de angiospermas

Famílias com uma espécie de angiosperma

Total de gêneros de angiospermas

Total de espécies do componente arbóreo

Total de espécies do componente arbustivo/subarbóreo

Espécies exclusivas do componente arbóreo

Espécies exclusivas do componente arbustivo/subarbóreo

Legenda
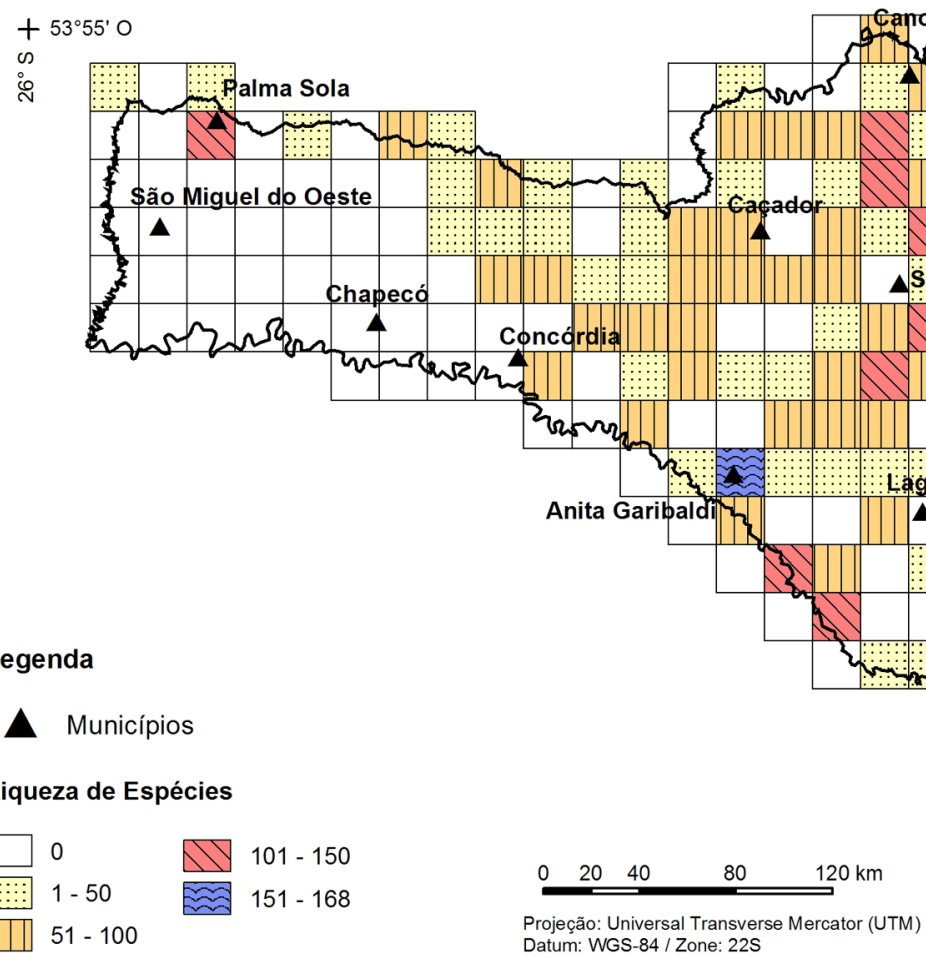

Figura 3 - Distribuição da riqueza de espécies registrada na Floresta Ombrófila Mista em Santa Catarina, baseada nas coletas feitas pelo Inventário Florístico Florestal de Santa Catarina.

Figure 3 - Species richness distribution, according to fertile material collected by Floristic Forest Inventory of Santa Catarina. 
Dentre as espécies exclusivas do componente arbustivo/subarbóreo, cabe destacar algumas espécies de sub-bosque de florestas com dossel mais fechado (Wasshausen \& Smith 1969; Legrand \& Klein 1969; Delprete et al. 2004): Justicia brasiliana, Ruellia angustiflora, Leandra dasytricha, Leandra regnellii, Miconia lymanii, Piper hispidum, Dahlstedtia pinnata, Psychotria nemorosa, Eugenia kleinii e Ossaea amygdaloides. Há também aquelas presentes em clareiras, áreas perturbadas ou bordas de florestas, não atingindo grandes tamanhos e, portanto, não presentes na sinúsia arbórea: Baccharis crispa, Baccharis dentata, Baccharis dracunculifolia e Berberis laurina (Mattos 1969; Barroso \& Bueno 2002).

Leite (2002) cita a ocorrência de 352 espécies arbóreas para a FOM, resultado levemente inferior ao obtido pelo presente estudo, que foi de 366 espécies. De acordo com o autor, dentre as espécies arbóreas que ocorrem na FOM, 13,3\% são exclusivas desta região fitoecológica, $45,7 \%$ são de ocorrência preferencial e outras $41 \%$ apresentam-se distribuídas preferencialmente nas demais regiões fitoecológicas.

No âmbito da FOM em Santa Catarina, Reitz et al. (1979) citam 194 espécies arbóreas para o planalto catarinense, sendo que destas, 157 foram amostradas pelo IFFSC. Baseado neste estudo de Reitz et al. (1979), 39 espécies citadas para a FOM de Santa Catarina não foram registradas pelo IFFSC (Tab. 2). Dentre estas, dez espécies foram amostradas pelo IFFSC somente na Floresta Ombrófila Densa e uma destas e duas outras constam do banco de dados SpeciesLink (2012) como coletadas na FOM de Santa Catarina nos últimos 20 anos, mas sem registros recentes (Tab. 2).

Em vista disso, torna-se imprescindível um novo esforço de coleta e, se possível, estudos populacionais, para averiguar o grau de vulnerabilidade dessas espécies, especialmente frente aos fatores atuais de perturbação da FOM. No entanto, essas 29 espécies estão com forte indicativo de ameaça, merecendo ações que visem ampliação do conhecimento e preservação. Ainda com relação a esse tema, as espécies Campomanesia hirsuta, Eugenia reitziana, Gochnatia velutina, Ocotea spectabilis e Symplocos oblongifolia foram citadas para Santa Catarina por Reitz et al. (1979), mas os especialistas contemporâneos destas famílias não confirmam esta informação. Como não há espécimes testemunhos citados na referida obra, não é possível confirmar estas citações e, segundo a Lista de espécies da flora do Brasil (2012), estas espécies não ocorrem neste estado.
À parte das considerações acerca dos levantamentos pretéritos na região da FOM, o fato atual de que, neste estudo, algumas espécies do componente arbóreo não tenham sido registradas no componente arbustivo/subarbóreo como indivíduos regenerantes, pode estar relacionado a uma série de fatores. Alguns deles poderiam levar à extinção local de suas populações, tais como a ausência de condições ecológicas favoráveis à sua instalação, possivelmente resultante do estágio sucessional da vegetação (Liebsch et al. 2008; Groeneveld et al. 2009), ou ainda a existência dos fatores de degradação constatados no interior dos fragmentos (Vibrans et al.2011). Outros fatores poderiam estar relacionados com problemas de amostragem, em virtude dos indivíduos não atenderem aos critérios de inclusão, ou ainda, o tamanho da área amostral ser insuficiente para abranger populações naturalmente pequenas e amplamente distribuídas, como por exemplo, aquelas que se distribuem preferencialmente em outras regiões fitoecológicas.

Além dos fatores supracitados, a roçada do subbosque e o deslocamento do gado para o interior dos remanescentes, técnica conhecida como invernada, agrava o desaparecimento de regenerantes de espécie arbóreas, como efeito do pastoreio ou mesmo aos danos físicos provocados pelo pisoteio no sub-bosque florestal (Sampaio \& Guarino 2007).

Acerca do levantamento florístico extra, realizado para complementar o registro das espécies nas áreas estudadas, o mesmo foi de extrema importância, uma vez que possibilitou grande incremento no número de registros, além de proporcionar a incorporação de 2.720 exsicatas ao Herbário FURB. Essa estratégia permitiu otimizar os recursos financeiros e de pessoal, além de resultar em ampliação do conhecimento da flora catarinense atual.

Apesar da evidente contribuição do levantamento florístico complementar para o registro das espécies que ocorrem na FOM em Santa Catarina, muitas áreas estão sem coletas (Fig. 3) pela ausência de florestas ou por se localizarem em outras regiões fitoecológicas não abrangidas no presente estudo (Floresta Ombrófila Densa, Floresta Estacional Decidual e Estepe Ombrófila) e, consequentemente, pela ausência de UAs implementadas. Esta diversidade por área de $400 \mathrm{~km}^{2}$ é considerada baixa, demonstrando a necessidade de maior esforço amostral para registrar a total biodiversidade da Flora de Santa Catarina. Destacamse as quadrículas situadas na região de Alfredo Wagner, Anita Garibaldi e Urubici, pela elevada 
Tabela 2 - Espécies arbóreas citadas por Reitz et al. (1979), para a Floresta Ombrófila Mista, e não encontradas no Inventário Florestal de Santa Catarina (IFFSC), nesta região fitoecológica. I - Representa outros registros da espécie, conforme segue: D - amostrada em Santa Catarina na Floresta Ombrófila Densa (FOD) ou C - com coleta registrada no CRIA nos últimos 20 anos.

Table 2 - Tree species cited by Reitz et al. (1979) to Mixed Ombrophyllous Forest and not found by the Forest Inventory of Santa Catarina (IFFSC) in this phytoecological region. I - Represents other records of the species, as follows: D - sampled in Santa Catarina in the Dense Rain Forest (FOD) or C - collection recorded in the last 20 years from CRIA.

\begin{tabular}{|c|c|c|c|}
\hline Família & Nome científico & Observações & $\mathbf{I}$ \\
\hline Apocynaceae & Aspidosperma ramiflorum Müll.Arg. & frequente & $\mathrm{D}$ \\
\hline \multirow[t]{2}{*}{ Aquifoliaceae } & Ilex chamaedryfolia Reissek & muito rara & \\
\hline & Ilex pseudobuxus Reissek & frequente & $\mathrm{D}, \mathrm{C}$ \\
\hline Asteraceae & Gochnatia velutina (Bong.) Cabrera & - & \\
\hline Elaeocarpaceae & Crinodendron brasiliense Reitz \& L.B.Sm. & típica de mata nebular & \\
\hline Erythroxylaceae & Erythroxylum argentinum O.E.Schulz & frequente & $\mathrm{C}$ \\
\hline Escalloniaceae & Escallonia farinacea A.St.-Hil. & - & \\
\hline Euphorbiaceae & Sebastiania schottiana (Müll.Arg.) Müll.Arg. & muito frequente & $\mathrm{C}$ \\
\hline Fabaceae & Mimosa taimbensis Burkart & endêmica dos Aparados da Serra & \\
\hline \multirow[t]{4}{*}{ Lauraceae } & Cinnamomum hatschbachii Vattimo-Gil & - & \\
\hline & Nectandra reticulata (Ruiz \& Pav.) Mez & - & \\
\hline & Ocotea acutifolia (Nees) Mez & frequente & \\
\hline & Ocotea spectabilis (Meisn.) Mez & - & \\
\hline Lythraceae & Lafoensia pacari A.St.-Hil. & rara & $\mathrm{D}$ \\
\hline Monimiaceae & Mollinedia elegans Tul. & rara & $\mathrm{D}$ \\
\hline \multirow[t]{13}{*}{ Myrtaceae } & Campomanesia adamantium (Cambess.) O.Berg & possivelmente muito rara & \\
\hline & Campomanesia aurea O.Berg & muito comum nos campos do planalto & \\
\hline & Campomanesia eugenioides (Cambess.) D.Legrand & muito rara & \\
\hline & Campomanesia hirsuta Gardner & - & \\
\hline & Eugenia blastantha (O.Berg) D.Legrand & & \\
\hline & Eugenia reitziana D.Legrand & raro & \\
\hline & Myrceugenia reitzii D.Legrand & rara & \\
\hline & Myrceugenia rufescens (DC.) D.Legrand \& Kausel & muito rara & $\mathrm{D}$ \\
\hline & Myrceugenia scutellata D.Legrand & muito rara & \\
\hline & Myrcia rupicola D.Legrand & rara & $\mathrm{D}$ \\
\hline & Psidium australe Cambess. & bastante rara & \\
\hline & Psidium grandifolium Mart. ex DC. & - & \\
\hline & Psidium salutare (Kunth) O.Berg & - & \\
\hline Nyctaginaceae & Bougainvillea glabra Choisy & rara e característica da FOD & $\mathrm{D}$ \\
\hline Proteaceae & Roupala asplenioides Sleumer & - & $\mathrm{D}$ \\
\hline Rhamnaceae & Colletia exserta Klotzsch ex Reissek & característica e exclusiva do planalto & \\
\hline \multirow[t]{2}{*}{ Rubiaceae } & Faramea porophylla (Vell.) Müll.Arg. & frequente & \\
\hline & Machaonia brasiliensis (Humb.) Cham. \& Schltdl. & - & \\
\hline Salicaceae & Prockia crucis P.Browne ex L. & - & $\mathrm{D}$ \\
\hline Solanaceae & Brunfelsia uniflora (Pohl) D.Don & característica e exclusiva do planalto & $\mathrm{D}$ \\
\hline \multirow[t]{3}{*}{ Symplocaceae } & Symplocos oblongifolia Casar. & - & \\
\hline & Symplocos pentandra (Mattos) Occhioni ex Aranha & muito rara & \\
\hline & Symplocos uniflora (Pohl) Benth. & - & \\
\hline
\end{tabular}


riqueza amostrada. Estas regiões são em geral compostas por mais de um tipo de formação, como no caso de Urubici que engloba o Morro da Igreja, ou seja, a Floresta Ombrófila Mista Altomontana e os Campos Naturais ou áreas de grande heterogeneidade geológica, como a Serra Geral.

Como apontado por Vibrans et al. (2011), $O$. porosa, O. odorifera e $A$. angustifolia ainda estão submetidas à pressão de uso. No entanto, o cruzamento dos dados qualitativos aqui apresentados com os quantitativos, ainda sob análise, poderão informar o grau de raridade em que se encontram as espécies da FOM, a exemplo do que foi efetuado por Caiafa \& Martins (2010), na porção meridional da Floresta Ombrófila Densa, e Fontana \& Sevegnani (2012), para a Floresta Estacional Decidual.

Com o registro de $43 \%$ e $22,7 \%$ das espécies citadas por Stehmann et al. (2009) e Forzza et al. (2010), respectivamente, o IFFSC amostrou um conjunto significativo das espécies da Floresta Atlântica em Santa Catarina e na região abrangida pela Floresta Ombrófila Mista. No entanto, muitas das espécies apareceram somente em uma ou poucas unidades amostrais, fato que torna preocupante sua manutenção nos ecossistemas. A riqueza concentrada em poucas famílias é uma característica comum em levantamentos similares, sendo também esperado que, na área de abrangência da Floresta Atlântica, haja um grande número de espécies concentrado em famílias como Asteraceae, Myrtaceae, Fabaceae, Solanaceae, Melastomataceae e Lauraceae.

Este mesmo estudo apontou uma tendência de concentração de riqueza nas áreas situadas no leste, principalmente nas proximidades das escarpas da Serra Geral. Estes dados, portanto, revestem-se de grande importância para a formulação de políticas públicas que visem à conservação in situ das espécies.

\section{Agradecimentos}

Este trabalho recebeu financiamento da Fundação de Amparo à Pesquisa e Inovação do Estado de Santa Catarina.

\section{Referências}

APG III - Angiosperm Phylogeny Group. 2009. An update of the Angiosperm Phylogeny Group classification for the orders and families of flowering plants: APG III. Botanical Journal of the Linnean Society 161: 105-121.

Backes, A. 2009. Distribuição geográfica atual da floresta com araucária: condicionamento climático. In: Fonseca, C.R.D.; Souza, A.F.; Leal-Zanchet, A.M.; Dutra, T.L.; Backes, A. \& Ganade G. (orgs.).
Floresta com araucária: ecologia, conservação e desenvolvimento sustentável. Holos, Ribeirão Preto. Pp. 39-44.

Barroso, G.M. \& Bueno, O. 2002. Compostas - 5. Subtribo: Baccharidinae. In: Reitz, R. (ed.). Flora Ilustrada Catarinense. Herbário Barbosa Rodrigues, Itajaí. Pp. 765-1065.

Caiafa, A.N. \& Martins, F.R. 2010. Forms of rarity of tree species in the southern Brazilian Atlantic rainforest. Biodiversity Conservation 19: 2597-2618.

Canhos, V.P.; Souza, S.; Giovanni, R.D. \& Canhos, D.A.L. 2004. Global biodiversity informatics: setting the scene for a "new world" of ecological modeling. Biodiversity Informatics 1: 1-13.

Christenhusz, M.J.M.; Reveal, J.L.; Farjon, A.; Gardner, M.F.; Mill, R.R. \& Chase, M.W. 2011. A new classification and linear sequence of extant gymnosperms. Phytotaxa 19: 55-70.

Citadini-Zanette, V.; Pereira, J.L.; Jarenkow, J.A.; Klein, A. S. \& Santos, R. 2011. Estrutura da sinúsia herbácea em Floresta Ombrófila Mista no Parque Nacional de Aparados da Serra, sul do Brasil. Revista Brasileira de Biociências 9: 56-63.

Cordeiro, J. \& Rodrigues, W.A. 2007. Caracterização fitossociológica de um remanescente de floresta ombrófila mista em Guarapuava, PR. Revista Árvore 31: 545-554.

Corona, P.; Chirici, G.; McRoberts, R.; Winter, S. \& Barbati, A. 2011. Contribution of large-scale forest inventories to biodiversity assessment and monitoring. Forest Ecology and Management 262: 2061-2069.

Delprete, P.G.; Smith, L.B. \& Klein, R.M. 2004. Rubiáceas. In: Reitz, R. (ed.). Flora ilustrada catarinense. Herbário Barbosa Rodrigues, Itajaí. 842p.

ESRI. 2011. ArcGis Desktop: Release 10. Environmwntal Systems Research Institute, Redlands.

Fontana, C. \& Sevegnani, L. 2012. Quais são as espécies arbóreas comuns da floresta estacional decidual em Santa Catarina? Revista de Estudos Ambientais 14: 74-88.

Formento, S.; Schorn, L.A. \& Ramos, R.A.B. 2004. Dinâmica estrutural arbórea de uma floresta ombrófila mista em Campo Belo do Sul, SC. Cerne 10: 196-212.

Forzza, R.C.; Baumgratz, J.F.A.; Bicudo, C.E.M.; Carvalho Jr., A.A.; Costa, A.; Costa, D.P.; Hopkins, M.; Leitman, P.M.; Lohmann, L.G.; Maia, L.C.; Martinelli, G.; Menezes, M.; Morim, M.P.; Nadruz Coelho, M.A.; Peixoto, A.L.; Pirani, J.R.; Prado, J.; Queiroz, L.P.; Souza, V.C.; Stehmann, J.R.; Sylvestre, L.S.; Walter, B.M.T. \& Zappi, D. 2010. Catálogo de plantas e fungos do Brasil. Instituto de Pesquisas Jardim Botânico do Rio de Janeiro, Rio de Janeiro. 1699p.

FURB - Herbário Dr. Roberto Miguel Klein. 2012. Herbário virtual da flora e dos fungos. Disponível em $<$ http://inct.splink.org.br>. Acesso em 15 Fev 2012. 
Gasper, A.L. 2012. Pteridófitas de Santa Catarina, Brasil: diversidade, distribuição geográfica e variáveis ambientais. Dissertação de Mestrado. Universidade Federal de Minas Gerais. 86p.

Groeneveld, J.; Alvesc, L.F.; Bernacci, L.C.; Catharino, E.L.M.; Knogge, C.; Metzger, J.P.; Pütza, S. \& Hutha, A. 2009. The impact of fragmentation and density regulation on forest succession in the Atlantic rain forest. Ecological Modelling 220: 2450-2459.

Guedes-Bruni, R.R.; Morin, M.P.; Lima, H.C. \& Sylvestre, L.S. 2002. Inventário florístico. In: Silvestre, L.S. \& Rosa, M.M.T. (eds.). Manual metodológico para estudos botânicos na Mata Atlântica. UFRRJ, Seropédica. 121p.

Hernandez, P.A.; Franke, I.; Herzog, S.K.; Pacheco, V.; Paniagua, L.; Quintana, H.L.; Soto, A.; Swenson, J.J.; Tovar, C.; Valqui, T.H.; Vargas, J. \& Young, B.E. 2008. Predicting species distributions in poorly-studied landscapes. Biodiversity and Conservation 17: 1353-1366.

Hijmans, R.J.; Cameron, S.E.; Parra, J.L.; Jones, P.G. \& Jarvis, A. 2005. Very high resolution interpolated climate surfaces for global land areas. International Journal of Climatology 25: 1965-1978.

Hopkins, M.J.G. 2007. Modelling the known and unknown plant biodiversity of the Amazon Basin. Journal of Biogeography 34: 1400-1411.

Hortal, J.; Lobo, J.M. \& Jiménez-Valverde, A. 2007 Limitations of biodiversity databases: case study on seed-plant diversity in Tenerife, Canary Islands. Conservation Biology 21: 853-63.

INCT. 2012. Herbário Virtual da Flora e dos Fungos. Disponível em $<$ http://inct.florabrasil.net/>. Acesso em 15 Fev 2012.

Klauberg, C.; Paludo, G.F.; Bortoluzzi, R.L.C. \& Mantovani, A. 2010. Florística e estrutura de um fragmento de floresta ombrófla mista no Planalto Catarinense. Biotemas 23: 35-47.

Klein, R.M. 1960. O aspecto dinâmico do pinheiro brasileiro. Sellowia 12: 17-44.

Klein, R.M. 1963. Observações e considerações sobre a vegetação do Planalto Nordeste catarinense. Sellowia 15: 39-56.

Klein, R.M. 1978. Mapa fitogeográfico do estado de Santa Catarina. In: Reitz, R. (ed.). Flora Ilustrada Catarinense. Herbário Barbosa Rodrigues, Itajaí. 24p.

Klein, R.M. 1984. Aspectos dinâmicos da vegetação do Sul do Brasil. Sellowia 36: 5-54.

Kreft, H. \& Jetz, W. 2007. Global patterns and determinants of vascular plant diversity. PNAS 104: 5925-5930.

Landrum, L.R. 1981. Amonograph of the genus Myrceugenia (Myrtaceae). Flora Neotropica 29: 1-135.

Legrand, C.D.J. \& Klein, R.M. 1969. Mirtáceas. In: Reitz, R. (ed.). Flora ilustrada catarinense. Herbário Barbosa Rodrigues, Itajaí. Pp. 45-216.
Leite, P.F. \& Klein, R.M. 1990. Geografia do Brasil. Vol. 2. IBGE, Rio de Janeiro. 420p.

Leite, P.F. 2002. Contribuição ao conhecimento fitoecológico do sul do Brasil. Ciência \& Ambiente 24: 51-63.

Liebsch, D.; Marques, M.C.M. \& Goldenberg, R. 2008. How long does the Atlantic Rain forest take to recover after a disturbance? Changes in species composition and ecological features during secondary succession. Biological Conservation 141: 1717-1725.

Lingner, D.V.; Oliveira, Y.M.M.D.; Rosot, N.C. \& Dlugosz, F.L. 2007. Caracterização da estrutura e da dinâmica de um remanescente de floresta de araucária no Planalto Catarinense. Pesquisa Florestal Brasileira 55: 55-66.

Forzza, R.C. et al. (eds.). 2012. Lista de espécies da flora do Brasil. Disponível em < http://floradobrasil.jbrj. gov.br/2012>. Acesso em 10 Fev 2012.

Martinelli, G. 2007. Mountain biodiversity in Brazil. Revista Brasileira de Botânica 30: 587-597.

Mattos, J.R. 1969. Berberidáceas. In: Reitz, R. (ed.). Flora Ilustrada Catarinense. Herbário Barbosa Rodrigues, Itajaí. 15p.

MMA - Ministério do Meio Ambiente. 2008. Espécies da flora brasileira ameaçadas de extinção. Instrução normativa n.6, de 23 de setembro de 2008.

Nascimento, A.R.T.; Longhi, S.J. \& Brena, D.A. 2001. Estrutura e padrões de distribuição espacial de espécies arbóreas em uma amostra de Floresta Ombrófila Mista em Nova Prata, RS. Ciência Florestal 11: 105-119.

Negrelle, R.A.B. \& Silva, F.C. 1992. Fitossociologia de um trecho de floresta com Araucaria angustifolia (Bert.) O. Ktze. no município de Caçador-SC. Boletim de Pesquisa Florestal 24: 37-54.

Pillar, V.D.; Müller, S.C.; Castilhos, Z. \& Jacques, A.V.A. (orgs.). 2009. Campos Sulinos: conservação e uso sustentável da biodiversidade. Ministério do Meio Ambiente, Brasília. 403p.

Randon Neto, R.M.; Watzlawick, L.F.; Caldeira, M.V.W. \& Schoeninger, E.R. 2002. Análise florística e estrutural de um fragmento de floresta ombrófila mista montana situado em Criúva, RS, Brasil. Ciência Florestal 12: 29-37.

Reitz, R.; Klein, R.M.; Reis, A. 1978. Projeto madeira de Santa Catarina. Sellowia 28: 11-320.

Reitz, R.; Klein, R.M.; Reis, A. 1979. Madeiras do Brasil. Lunardelli, Florianópolis. 320p.

Reitz. R. 1965. Plano de coleção. In: Reitz, R. (ed.). Flora Ilustrada Catarinense. Herbário Barbosa Rodrigues, Itajaí. 71p.

Rivers, M.C.; Bachman, S.P.; Meagher, T.R.; Nic Lughadha, E. \& Brummitt, N.A. 2010. Subpopulations, locations and fragmentation: applying IUCN red list criteria to herbarium specimen data. Biodiversity and Conservation 19: 2071-2085. 
Sampaio, M.B. \& Guarino, E.S.G. 2007. Efeitos do pastoreio de bovinos na estrutura populacional de plantas em fragmentos de floresta ombrófila mista. Revista Árvore 31: 1035-1046.

Schatz, G.E. 2002. Taxonomy and herbaria in service of plant conservation: lessons from Madagascar's endemic families. Annals of the Missouri Botanical Garden 89: 145-152.

Sobral, M. \& Stehmann, J.R. 2009. An analysis of new angiosperm species discoveries in Brazil (19902006). Taxon 58: 227-232.

Sobral, M. 2003. A família das Myrtaceae no Rio Grande do Sul. São Leopoldo, Unisinos, 215p.

Species Link. 2012. Base de dados eletrônica. Disponível em $<$ http://www.splink.org.br>. Acesso em 10 Fev 2012.

Stehmann, J.R.; Forzza, R.C.; Salino, A.; Sobral, M.; Costa, D.P. \& Kamino, L.H.Y. 2009. Plantas da Floresta Atlântica. Instituto de Pesquisas Jardim Botânico do Rio de Janeiro, Rio de Janeiro. 516p.

Thiers, B. 2012. Index Herbariorum: a global directory of public herbaria and associated staff. New York Botanical Garden's Virtual Herbarium. Disponível em $<$ http://sweetgum.nybg.org/ih/>. Acesso em 1 Fev 2012.

Vibrans, A.C.; Sevegnani, L.; Lingner, D.V.; Gasper, A.L. \& Sabbagh, S. 2010. Inventário Florístico Florestal de Santa Catarina (IFFSC): aspectos metodológicos e operacionais. Pesquisa Florestal Brasileira 30: 291-302.

Vibrans, A.C.; McRoberts, E.; Moser, P. \& Nicoletti, A.L. 2013. Using satellite image-based maps and ground inventory data to estimate the area of the remaining Atlantic forest in the Brazilian state of Santa Catarina. Remote Sensing of Environment 130: 87-95.

Vibrans, A.C.; Sevegnani, L.; Uhlmann, A.; Schorn, L.A.; Sobral, M.; Gasper, A.L.; Lingner, D.V.; Brogni, E.; Klemz, G.; Godoy, M.B. \& Verdi, M. 2011. Structure of mixed ombrophyllous forests with Araucaria angustifolia (Araucariaceae) under external stress in Southern Brazil. Revista de Biologia Tropical 59: 1371-1387.

Wasshausen, D.C. \& Smith, L.B. 1969. Acantáceas. In: Reitz, R. (ed.). Flora Ilustrada Catarinense. Herbário Barbosa Rodrigues, Itajaí. 134 p.

Werneck, M.S.; Sobral, M.; Rocha, C.T.V. \& Landau, E.C. 2011. Distribution and endemism of angiosperms in the Atlantic forest. Natureza \& Conservação 9: 188-193.

Yesson, C.; Brewer, P.W.; Sutton, T.; Caithness, N.; Pahwa, J.S.; Burgess, M.; Gray, W.A.; White, R.J.; Jones, A.C.; Bisby, F.A. \& Culham, A. 2007. How global is the global biodiversity information facility? PLoS One 2: e1124. 